Acta Crystallographica Section E

Structure Reports

Online

ISSN 1600-5368

\section{Aquachloridobis(1,10-phenanthroline- $\left.\boldsymbol{\kappa}^{2} N, N^{\prime}\right)$ zinc(II) chloride $N, N$-dimethyl- formamide solvate}

\author{
Li-Li Kong, ${ }^{a}$ Shan Gao, ${ }^{a}$ Li-Hua Huo ${ }^{a}$ and Seik Weng $\mathrm{Ng}^{\mathrm{b}}$ *

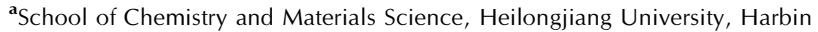 \\ 150080, People's Republic of China, and 'bepartment of Chemistry, University of \\ Malaya, 50603 Kuala Lumpur, Malaysia \\ Correspondence e-mail: seikweng@um.edu.my
}

Received 7 January 2008; accepted 21 January 2008

Key indicators: single-crystal X-ray study; $T=295 \mathrm{~K}$; mean $\sigma(\mathrm{C}-\mathrm{C})=0.004 \AA$; disorder in solvent or counterion; $R$ factor $=0.033 ; w R$ factor $=0.103$; data-toparameter ratio $=15.4$.

The $\mathrm{Zn}$ atom in the title salt, $\left[\mathrm{ZnCl}\left(\mathrm{C}_{12} \mathrm{H}_{8} \mathrm{~N}_{2}\right)_{2}\left(\mathrm{H}_{2} \mathrm{O}\right)\right] \mathrm{Cl} \cdot-$ $\mathrm{C}_{3} \mathrm{H}_{7} \mathrm{NO}$, is chelated by two phenanthroline molecules and is bonded to one chloride ion and one water molecule, resulting in a $\mathrm{ZnN}_{4} \mathrm{ClO}$ octahedral coordination environment with the $\mathrm{Cl}$ and $\mathrm{O}$ atoms in a cis conformation. The cations and anions are linked by $\mathrm{O}-\mathrm{H} \cdots \mathrm{Cl}$ hydrogen bonds across a center of inversion, forming a hydrogen-bonded dimeric association. The dimethylformamide solvent molecule is disordered over two orientations in a $0.56(1): 0.44$ (1) ratio.

\section{Related literature}

The title compound is isostructural with the cobalt and nickel analogs: see Liu, Gao, Huo \& Ng (2004); Liu, Liu \& Zhong (2004).

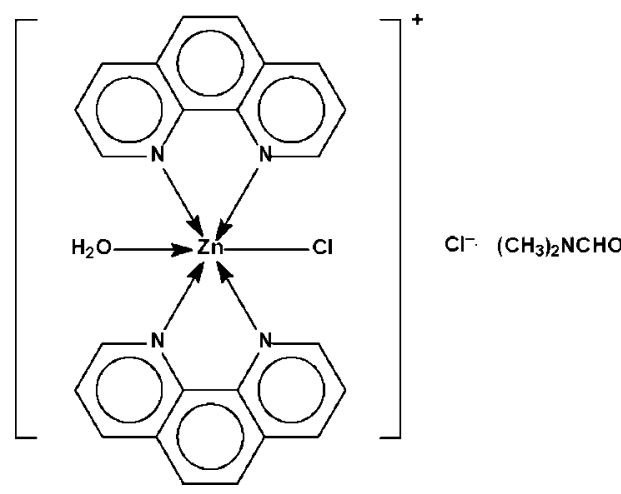

\section{Experimental}

\section{Crystal data}

$\begin{array}{ll}{\left[\mathrm{ZnCl}\left(\mathrm{C}_{12} \mathrm{H}_{8} \mathrm{~N}_{2}\right)_{2}\left(\mathrm{H}_{2} \mathrm{O}\right)\right] \mathrm{Cl} \cdot-} & a=9.6743(3) \AA \\ \mathrm{C}_{3} \mathrm{H}_{7} \mathrm{NO} & b=11.6096(5) \AA \\ M_{r}=587.79 & c=12.7486(5) \AA \\ \text { Triclinic, } P \overline{1} & \alpha=67.004(1)^{\circ}\end{array}$

$$
\begin{aligned}
& \beta=85.995(1)^{\circ} \\
& \gamma=80.025(1)^{\circ} \\
& V=1298.14(9) \AA^{3} \\
& Z=2
\end{aligned}
$$

\author{
Mo $K \alpha$ radiation \\ $\mu=1.19 \mathrm{~mm}^{-1}$ \\ $T=295$ (2) K \\ $0.30 \times 0.24 \times 0.18 \mathrm{~mm}$
}

\section{Data collection}

Rigaku R-AXIS RAPID diffractometer

Absorption correction: multi-scan (ABSCOR; Higashi, 1995)

$T_{\min }=0.660, T_{\max }=0.815$

Refinement

$R\left[F^{2}>2 \sigma\left(F^{2}\right)\right]=0.033$

$w R\left(F^{2}\right)=0.103$

$S=1.11$

5884 reflections

383 parameters

61 restraints

12819 measured reflections 5884 independent reflections 4602 reflections with $I>2 \sigma(I)$ $R_{\text {int }}=0.022$

$\mathrm{H}$ atoms treated by a mixture of independent and constrained refinement

$\Delta \rho_{\max }=0.43{\mathrm{e} \AA^{-3}}^{-3}$

$\Delta \rho_{\min }=-0.36{\mathrm{e} \AA^{-3}}^{-3}$

Table 1

Selected bond lengths ( $\mathrm{A})$.

\begin{tabular}{llll}
\hline $\mathrm{Zn} 1-\mathrm{N} 1$ & $2.190(2)$ & $\mathrm{Zn} 1-\mathrm{N} 4$ & $2.275(2)$ \\
$\mathrm{Zn} 1-\mathrm{N} 2$ & $2.198(2)$ & $\mathrm{Zn} 1-\mathrm{O} 1 w$ & $2.090(2)$ \\
$\mathrm{Zn} 1-\mathrm{N} 3$ & $2.157(2)$ & $\mathrm{Zn} 1-\mathrm{Cl} 1$ & $2.3520(6)$ \\
\hline
\end{tabular}

Table 2

Hydrogen-bond geometry $\left(\AA{ }^{\circ}\right)$.

\begin{tabular}{lllll}
\hline$D-\mathrm{H} \cdots A$ & $D-\mathrm{H}$ & $\mathrm{H} \cdots A$ & $D \cdots A$ & $D-\mathrm{H} \cdots A$ \\
\hline $\mathrm{O} 1 w-\mathrm{H} 1 w 1 \cdots \mathrm{Cl} 2$ & $0.85(3)$ & $2.29(3)$ & $3.112(2)$ & $163(3)$ \\
$\mathrm{O} 1 w-\mathrm{H} 1 w 2 \cdots \mathrm{Cl} 2{ }^{\mathrm{i}}$ & $0.84(3)$ & $2.24(3)$ & $3.079(2)$ & $172(3)$ \\
\hline
\end{tabular}

Symmetry code: (i) $-x+1,-y+2,-z+1$.

Data collection: RAPID-AUTO (Rigaku, 1998); cell refinement: RAPID-AUTO; data reduction: CrystalStructure (Rigaku/MSC, 2002); method used to solve structure: atomic coordinates taken from the iostructural Co compound (Liu et al., 2004); program(s) used to refine structure: SHELXL97 (Sheldrick, 2008); molecular graphics: $X$-SEED (Barbour, 2001); software used to prepare material for publication: publCIF (Westrip, 2008).

We thank the Heilongjiang Province Natural Science Foundation (No. B200501), the Scientific Fund for Remarkable Teachers of Heilongjiang Province (No. 1054 G036), Heilongjiang University and the University of Malaya for supporting this work.

Supplementary data and figures for this paper are available from the IUCr electronic archives (Reference: HB2689).

\title{
References
}

Barbour, L. J. (2001). J. Supramol. Chem. 1, 189-191.

Higashi, T. (1995). ABSCOR. Rigaku Corporation, Tokyo, Japan.

Liu, J.-W., Gao, S., Huo, L.-H. \& Ng, S. W. (2004). Acta Cryst. E60, m501-m503.

Liu, H., Liu, L.-P. \& Zhong, B.-H. (2004). Anal. Sci. 20, x63-x64.

Rigaku (1998). RAPID-AUTO. Rigaku Corporation, Tokyo, Japan.

Rigaku/MSC (2002). CrystalStructure. Rigaku/MSC, The Woodlands, Texas, USA.

Sheldrick, G. M. (2008). Acta Cryst. A64, 112-122.

Westrip, S. P. (2008). publCIF. In preparation. 


\section{supporting information}

Acta Cryst. (2008). E64, m423 [doi:10.1107/S1600536808002237]

\section{Aquachloridobis $\left(1,10\right.$-phenanthroline- $\left.\kappa^{2} N, N^{\prime}\right)$ zinc(II) chloride $N, N$-dimethyl- formamide solvate}

\section{Li-Li Kong, Shan Gao, Li-Hua Huo and Seik Weng Ng}

\section{S1. Comment}

The title compound, (I), is isostructural with its cobalt (Liu, Gao, Huo \& Ng, 2004) and nickel analogs (Liu, Liu \& Zhong, 2004).

The $\mathrm{Zn}$ atom in (I) is chelated by two phenanthroline molecules and bonded to one chloride ion and one water molecule, resulting in a $\mathrm{ZnN}_{4} \mathrm{ClO}$ octahedral coordination environment with the $\mathrm{Cl}$ and $\mathrm{O}$ atoms in a cis conformation (Table 1, Fig. 1). The cations and anions are linked by $\mathrm{O}-\mathrm{H} \cdots \mathrm{Cl}$ hydrogen bonds across a center of inversion forming a hydrogenbonded dimeric association. Details of the hydrogen bonds are given in Table 2.

\section{S2. Experimental}

Zinc diacetate dihydrate $(1 \mathrm{mmol})$ and 1,10-phenanthroline $(2 \mathrm{mmol})$ were dissolved in a DMF-water mixture. Several drops of hydrochloric acid were added, and the mixture set aside for the growth of crystals.. The mixture was filtered and colorless blocks of (I) were isolated after several days.

\section{S3. Refinement}

The carbon-bound $\mathrm{H}$ atoms were placed in calculated positions $(\mathrm{C}-\mathrm{H}=0.93-0.97 \AA)$ and refined as riding with $U_{\text {iso }}(\mathrm{H})$ $1.2-1.5 U_{\text {eq }}(\mathrm{C})$.

The water $\mathrm{H}$-atoms were located in a difference map, and were refined with a distance restraint of $\mathrm{O}-\mathrm{H}=0.85 \pm 0.01 \AA$; their $U_{\text {iso }}$ values were refined.

The DMF solvate molecule is disordered but the disorder components share a nitrogen atom. The $\mathrm{C}-\mathrm{O}$ distance was restrained to $1.25 \pm 0.01 \AA$, the $N-\mathrm{C}_{\text {carbonyl }}$ distance to $1.35 \pm 0.01 \AA$ and the $N-\mathrm{C}_{\text {methyl }}$ distance to $1.45 \pm 0.01 \AA$. The molecule was restrained to be nearly flat. The occupations of the disorder components refined to 0.56 (1):0.44 (1). 


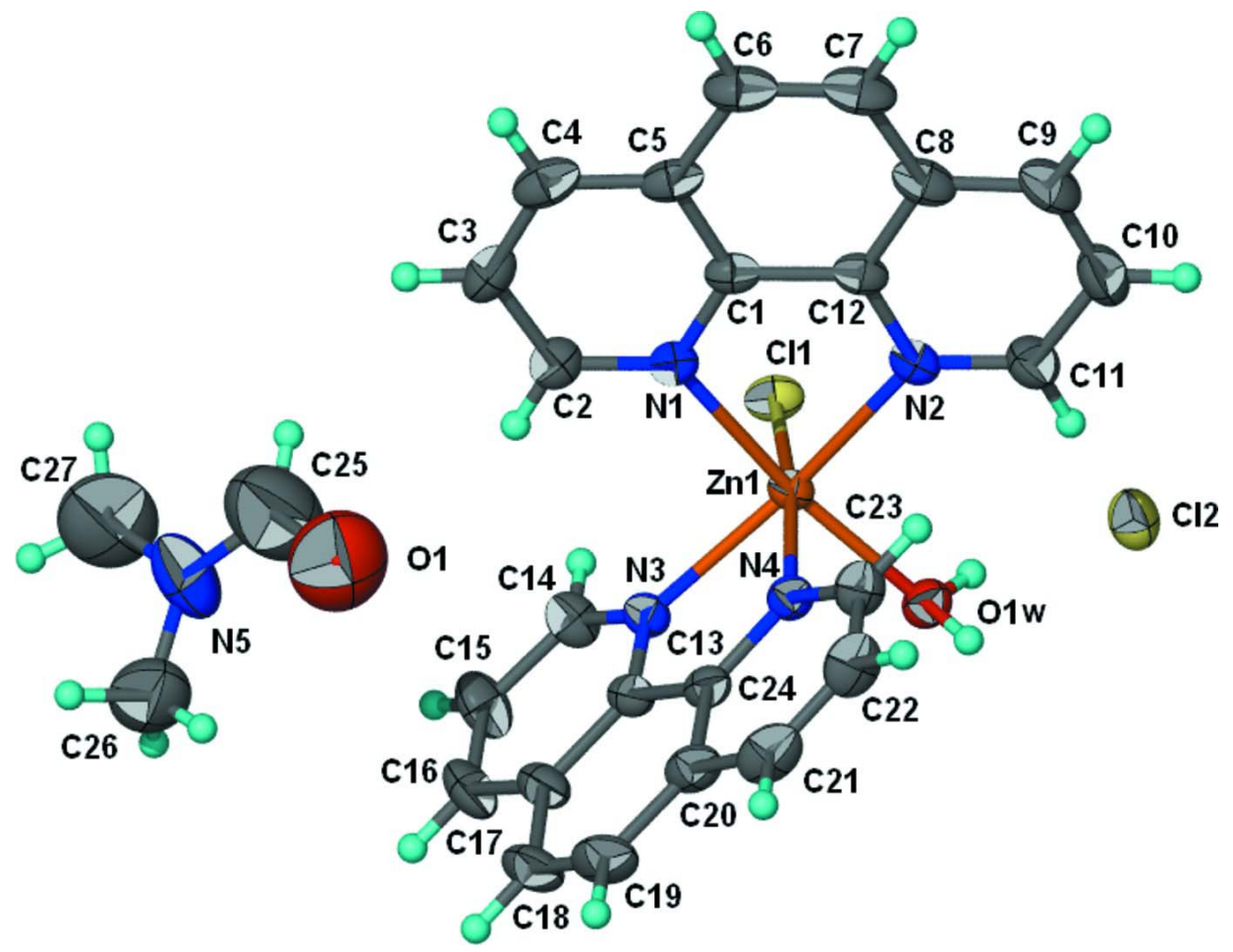

\section{Figure 1}

View of the molecular structure of (I); displacement ellipsoids are drawn at the $50 \%$ probability level, and $\mathrm{H}$ atoms as spheres of arbitrary radius. Only one disorder component of the DMF molecule is shown.

Aquachloridobis(1,10-phenanthroline- $\left.\kappa^{2} N, N^{\prime}\right)$ zinc(II) chloride $N, N$-dimethylformamide solvate

\section{Crystal data}

$\left[\mathrm{ZnCl}\left(\mathrm{C}_{12} \mathrm{H}_{8} \mathrm{~N}_{2}\right)_{2}\left(\mathrm{H}_{2} \mathrm{O}\right)\right] \mathrm{Cl} \cdot \mathrm{C}_{3} \mathrm{H}_{7} \mathrm{NO}$

$M_{r}=587.79$

Triclinic, $P \overline{1}$

Hall symbol: -P 1

$a=9.6743(3) \AA$

$b=11.6096(5) \AA$

$c=12.7486(5) \AA$

$\alpha=67.004(1)^{\circ}$

$\beta=85.995(1)^{\circ}$

$\gamma=80.025(1)^{\circ}$

$V=1298.14(9) \AA^{3}$

\section{Data collection}

Rigaku R-AXIS RAPID

diffractometer

Radiation source: fine-focus sealed tube

Graphite monochromator

Detector resolution: 10.000 pixels $\mathrm{mm}^{-1}$

$\omega$ scans

Absorption correction: multi-scan

(ABSCOR; Higashi, 1995)

$T_{\min }=0.660, T_{\max }=0.815$
$Z=2$

$F(000)=604$

$D_{\mathrm{x}}=1.504 \mathrm{Mg} \mathrm{m}^{-3}$

Mo $K \alpha$ radiation, $\lambda=0.71073 \AA$

Cell parameters from 10359 reflections

$\theta=3.1-27.5^{\circ}$

$\mu=1.19 \mathrm{~mm}^{-1}$

$T=295 \mathrm{~K}$

Block, colorless

$0.30 \times 0.24 \times 0.18 \mathrm{~mm}$

12819 measured reflections

5884 independent reflections

4602 reflections with $I>2 \sigma(I)$

$R_{\text {int }}=0.022$

$\theta_{\text {max }}=27.5^{\circ}, \theta_{\min }=3.1^{\circ}$

$h=-12 \rightarrow 12$

$k=-15 \rightarrow 15$

$l=-15 \rightarrow 16$ 


\section{Refinement}

Refinement on $F^{2}$

Least-squares matrix: full

$R\left[F^{2}>2 \sigma\left(F^{2}\right)\right]=0.033$

$w R\left(F^{2}\right)=0.103$

$S=1.11$

5884 reflections

383 parameters

61 restraints

Primary atom site location: structure-invariant direct methods
Secondary atom site location: difference Fourier map

Hydrogen site location: inferred from neighbouring sites

$\mathrm{H}$ atoms treated by a mixture of independent and constrained refinement

$w=1 /\left[\sigma^{2}\left(F_{0}^{2}\right)+(0.0615 P)^{2}\right]$ where $P=\left(F_{\mathrm{o}}^{2}+2 F_{\mathrm{c}}{ }^{2}\right) / 3$

$(\Delta / \sigma)_{\max }=0.001$

$\Delta \rho_{\max }=0.43 \mathrm{e} \AA^{-3}$

$\Delta \rho_{\min }=-0.36$ e $\AA^{-3}$

Fractional atomic coordinates and isotropic or equivalent isotropic displacement parameters $\left(\AA^{2}\right)$

\begin{tabular}{|c|c|c|c|c|c|}
\hline & $x$ & $y$ & $z$ & $U_{\text {iso }} * / U_{\text {eq }}$ & Occ. $(<1)$ \\
\hline Zn1 & $0.69100(2)$ & $0.67147(2)$ & $0.71925(2)$ & $0.02999(10)$ & \\
\hline $\mathrm{Cl1}$ & $0.57695(6)$ & $0.68729(6)$ & $0.88327(5)$ & $0.04014(15)$ & \\
\hline $\mathrm{Cl} 2$ & $0.39933(7)$ & $1.05103(6)$ & $0.64297(5)$ & $0.04871(17)$ & \\
\hline $\mathrm{O} 1 \mathrm{~W}$ & $0.53618(17)$ & $0.80418(17)$ & $0.61027(14)$ & 0.0387 (4) & \\
\hline H1W1 & $0.498(3)$ & $0.861(2)$ & $0.634(2)$ & $0.059(9)^{*}$ & \\
\hline H1W2 & $0.553(3)$ & $0.837(3)$ & $0.5398(10)$ & $0.055(8)^{*}$ & \\
\hline N1 & $0.88448(17)$ & $0.55649(18)$ & $0.80695(15)$ & $0.0324(4)$ & \\
\hline $\mathrm{N} 2$ & 0.83314 (19) & 0.80984 (18) & $0.68954(16)$ & 0.0352 (4) & \\
\hline $\mathrm{N} 3$ & $0.61286(18)$ & $0.50502(17)$ & $0.72752(15)$ & $0.0316(4)$ & \\
\hline N4 & $0.78178(18)$ & $0.63714(18)$ & $0.56257(15)$ & 0.0317 (4) & \\
\hline N5 & 0.7617 (3) & 0.0237 (3) & $0.9642(3)$ & $0.0874(10)$ & \\
\hline $\mathrm{C} 1$ & $0.9880(2)$ & $0.6243(2)$ & $0.80178(18)$ & $0.0324(5)$ & \\
\hline $\mathrm{C} 2$ & $0.9080(2)$ & $0.4331(2)$ & $0.8658(2)$ & $0.0402(5)$ & \\
\hline $\mathrm{H} 2$ & 0.8381 & 0.3861 & 0.8686 & $0.048^{*}$ & \\
\hline $\mathrm{C} 3$ & $1.0332(3)$ & $0.3698(3)$ & $0.9242(2)$ & $0.0464(6)$ & \\
\hline $\mathrm{H} 3$ & 1.0454 & 0.2826 & 0.9651 & $0.056^{*}$ & \\
\hline $\mathrm{C} 4$ & $1.1368(3)$ & $0.4364(3)$ & 0.9207 (2) & $0.0481(6)$ & \\
\hline $\mathrm{H} 4$ & 1.2201 & 0.3953 & 0.9601 & $0.058^{*}$ & \\
\hline $\mathrm{C} 5$ & 1.1177 (2) & $0.5674(3)$ & $0.8576(2)$ & $0.0400(6)$ & \\
\hline C6 & $1.2211(2)$ & $0.6458(3)$ & $0.8478(2)$ & $0.0509(7)$ & \\
\hline H6 & 1.3063 & 0.6092 & 0.8855 & $0.061 *$ & \\
\hline C7 & $1.1979(3)$ & $0.7700(3)$ & $0.7860(3)$ & $0.0532(7)$ & \\
\hline $\mathrm{H} 7$ & 1.2687 & 0.8175 & 0.7786 & $0.064^{*}$ & \\
\hline $\mathrm{C} 8$ & $1.0664(2)$ & $0.8316(3)$ & $0.7308(2)$ & $0.0439(6)$ & \\
\hline C9 & $1.0341(3)$ & $0.9631(3)$ & $0.6682(2)$ & $0.0530(7)$ & \\
\hline H9 & 1.1007 & 1.0150 & 0.6596 & $0.064 *$ & \\
\hline $\mathrm{C} 10$ & $0.9053(3)$ & $1.0136(3)$ & $0.6203(3)$ & $0.0553(7)$ & \\
\hline H10 & 0.8825 & 1.1005 & 0.5799 & $0.066^{*}$ & \\
\hline C11 & $0.8071(3)$ & $0.9338(2)$ & $0.6322(2)$ & $0.0456(6)$ & \\
\hline H11 & 0.7196 & 0.9696 & 0.5984 & $0.055^{*}$ & \\
\hline $\mathrm{C} 12$ & 0.9604 (2) & 0.7587 (2) & $0.73849(18)$ & $0.0339(5)$ & \\
\hline $\mathrm{C} 13$ & 0.6459 (2) & 0.4679 (2) & $0.63922(19)$ & $0.0324(5)$ & \\
\hline C14 & $0.5353(2)$ & $0.4368(2)$ & $0.8115(2)$ & $0.0421(6)$ & \\
\hline
\end{tabular}




\begin{tabular}{|c|c|c|c|c|c|}
\hline H14 & 0.5150 & 0.4599 & 0.8737 & $0.050^{*}$ & \\
\hline $\mathrm{C} 15$ & 0.4828 (3) & $0.3327(3)$ & 0.8111 & $0.0546(7)$ & \\
\hline H15 & 0.4287 & 0.2878 & 0.8716 & $0.065^{*}$ & \\
\hline $\mathrm{C} 16$ & $0.5120(3)$ & $0.2975(3)$ & $0.7202(3)$ & $0.0527(7)$ & \\
\hline H16 & 0.4758 & 0.2295 & 0.7175 & $0.063 *$ & \\
\hline $\mathrm{C} 17$ & $0.5967(2)$ & $0.3644(2)$ & $0.6314(2)$ & $0.0415(6)$ & \\
\hline $\mathrm{C} 18$ & $0.6358(3)$ & $0.3313(3)$ & $0.5352(2)$ & $0.0525(7)$ & \\
\hline H18 & 0.6026 & 0.2636 & 0.5291 & $0.063 *$ & \\
\hline C19 & $0.7204(3)$ & $0.3973(3)$ & $0.4532(2)$ & $0.0541(7)$ & \\
\hline H19 & 0.7447 & 0.3740 & 0.3916 & $0.065^{*}$ & \\
\hline $\mathrm{C} 20$ & $0.7732(2)$ & $0.5017(3)$ & $0.4589(2)$ & $0.0417(5)$ & \\
\hline $\mathrm{C} 21$ & $0.8641(3)$ & $0.5729(3)$ & $0.3765(2)$ & $0.0530(7)$ & \\
\hline $\mathrm{H} 21$ & 0.8931 & 0.5519 & 0.3142 & $0.064 *$ & \\
\hline $\mathrm{C} 22$ & $0.9091(3)$ & $0.6714(3)$ & $0.3882(2)$ & $0.0501(7)$ & \\
\hline $\mathrm{H} 22$ & 0.9683 & 0.7188 & 0.3341 & $0.060^{*}$ & \\
\hline $\mathrm{C} 23$ & $0.8649(2)$ & $0.7005(2)$ & $0.4829(2)$ & $0.0412(5)$ & \\
\hline $\mathrm{H} 23$ & 0.8960 & 0.7686 & 0.4899 & $0.049 *$ & \\
\hline $\mathrm{C} 24$ & $0.7357(2)$ & $0.5379(2)$ & $0.55131(18)$ & $0.0322(5)$ & \\
\hline $\mathrm{O} 1$ & $0.8615(13)$ & $0.1721(13)$ & $0.8303(11)$ & $0.145(5)$ & $0.560(9)$ \\
\hline $\mathrm{C} 25$ & $0.8320(9)$ & $0.1203(9)$ & $0.9327(10)$ & $0.153(5)$ & $0.560(9)$ \\
\hline $\mathrm{H} 25$ & 0.8587 & 0.1486 & 0.9860 & $0.184^{*}$ & $0.560(9)$ \\
\hline $\mathrm{C} 26$ & $0.7138(10)$ & $-0.0335(9)$ & $0.8953(7)$ & $0.105(3)$ & $0.560(9)$ \\
\hline H26A & 0.7384 & 0.0105 & 0.8171 & $0.157^{*}$ & $0.560(9)$ \\
\hline $\mathrm{H} 26 \mathrm{~B}$ & 0.6137 & -0.0287 & 0.9020 & $0.157^{*}$ & $0.560(9)$ \\
\hline $\mathrm{H} 26 \mathrm{C}$ & 0.7571 & -0.1207 & 0.9204 & $0.157^{*}$ & $0.560(9)$ \\
\hline $\mathrm{C} 27$ & $0.7266(10)$ & $-0.0410(11)$ & $1.0865(6)$ & $0.137(4)$ & $0.560(9)$ \\
\hline $\mathrm{H} 27 \mathrm{~A}$ & 0.8004 & -0.0406 & 1.1329 & $0.206^{*}$ & $0.560(9)$ \\
\hline H27B & 0.7166 & -0.1269 & 1.1017 & $0.206^{*}$ & $0.560(9)$ \\
\hline $\mathrm{H} 27 \mathrm{C}$ & 0.6401 & 0.0027 & 1.1039 & $0.206^{*}$ & $0.560(9)$ \\
\hline $\mathrm{O} 1^{\prime}$ & 0.9009 (13) & $0.1667(11)$ & $0.8546(11)$ & $0.115(4)$ & $0.440(9)$ \\
\hline $\mathrm{C} 25^{\prime}$ & $0.8264(10)$ & $0.0799(9)$ & $0.8675(9)$ & $0.100(4)$ & $0.440(9)$ \\
\hline $\mathrm{H} 25^{\prime}$ & 0.8180 & 0.0563 & 0.8067 & $0.120^{*}$ & $0.440(9)$ \\
\hline $\mathrm{C} 26^{\prime}$ & $0.7784(13)$ & $0.0665(13)$ & $1.0524(9)$ & $0.135(5)$ & $0.440(9)$ \\
\hline $\mathrm{H} 26 \mathrm{D}$ & 0.8736 & 0.0797 & 1.0528 & $0.202 *$ & $0.440(9)$ \\
\hline $\mathrm{H} 26 \mathrm{E}$ & 0.7569 & 0.0040 & 1.1246 & $0.202 *$ & $0.440(9)$ \\
\hline $\mathrm{H} 26 \mathrm{~F}$ & 0.7161 & 0.1447 & 1.0393 & $0.202 *$ & $0.440(9)$ \\
\hline $\mathrm{C} 27^{\prime}$ & $0.6808(12)$ & $-0.0719(10)$ & $0.9728(17)$ & $0.168(7)$ & $0.440(9)$ \\
\hline H27D & 0.7428 & -0.1478 & 0.9772 & $0.253 *$ & $0.440(9)$ \\
\hline $\mathrm{H} 27 \mathrm{E}$ & 0.6220 & -0.0425 & 0.9069 & $0.253^{*}$ & $0.440(9)$ \\
\hline $\mathrm{H} 27 \mathrm{~F}$ & 0.6234 & -0.0895 & 1.0401 & $0.253 *$ & $0.440(9)$ \\
\hline
\end{tabular}

Atomic displacement parameters $\left(\AA^{2}\right)$

\begin{tabular}{lllllll}
\hline & $U^{11}$ & $U^{22}$ & $U^{33}$ & $U^{12}$ & $U^{13}$ & $U^{23}$ \\
\hline Zn1 & $0.02905(14)$ & $0.03305(16)$ & $0.03096(15)$ & $-0.00828(10)$ & $0.00059(10)$ & $-0.01436(11)$ \\
C11 & $0.0364(3)$ & $0.0549(4)$ & $0.0340(3)$ & $-0.0079(3)$ & $0.0019(2)$ & $-0.0225(3)$ \\
C12 & $0.0604(4)$ & $0.0398(3)$ & $0.0463(4)$ & $-0.0088(3)$ & $0.0078(3)$ & $-0.0180(3)$ \\
O1W & $0.0421(9)$ & $0.0380(10)$ & $0.0353(9)$ & $-0.0009(7)$ & $-0.0029(7)$ & $-0.0151(8)$
\end{tabular}


supporting information

\begin{tabular}{|c|c|c|c|c|c|c|}
\hline N1 & $0.0296(8)$ & $0.0378(10)$ & $0.0310(9)$ & $-0.0059(8)$ & 0.0007 (7) & $-0.0146(8)$ \\
\hline $\mathrm{N} 2$ & $0.0348(9)$ & $0.0380(11)$ & $0.0381(10)$ & $-0.0104(8)$ & $0.0016(8)$ & $-0.0187(9)$ \\
\hline N3 & $0.0311(9)$ & $0.0338(10)$ & $0.0328(10)$ & -0.0085 & $0.0026(7)$ & $-0.0149(8)$ \\
\hline N4 & $0.0330(9)$ & $0.0334(10)$ & $0.0288(9)$ & $-0.0045(8)$ & $0.0006(7)$ & $-0.0126(8)$ \\
\hline N5 & $0.106(2)$ & $0.069(2)$ & $0.099(2)$ & $-0.0437(19)$ & $0.034(2)$ & $-0.0382(18)$ \\
\hline $\mathrm{C} 1$ & $0.0259(9)$ & $0.0473(13)$ & $0.0299(11)$ & $-0.0061(9)$ & $0.0024(8)$ & $-0.0216(10)$ \\
\hline $\mathrm{C} 2$ & $0.0375(11)$ & 0.0439 (14) & $0.0372(13)$ & $-0.0055(10)$ & $0.0016(10)$ & $-0.0140(11)$ \\
\hline $\mathrm{C} 3$ & $0.0460(13)$ & $0.0473(15)$ & $0.0370(13)$ & 0.0054 (11) & $-0.0050(10)$ & $-0.0111(11)$ \\
\hline $\mathrm{C} 4$ & $0.0369(12)$ & $0.0707(19)$ & $0.0369(13)$ & $0.0060(12)$ & $-0.0041(10)$ & -0.0263 \\
\hline $\mathrm{C} 5$ & $0.0291(10)$ & $0.0619(17)$ & $0.0347(12)$ & $-0.0006(11)$ & $-0.0019(9)$ & $-0.0272(12)$ \\
\hline C6 & $0.0282(11)$ & $0.081(2)$ & $0.0568(17)$ & $-0.0059(12)$ & $-0.0015(11)$ & $-0.0421(16)$ \\
\hline $\mathrm{C} 7$ & $0.0324(12)$ & $0.080(2)$ & $0.0638(18)$ & $-0.0224(13)$ & $0.0055(12)$ & $-0.0414(17)$ \\
\hline $\mathrm{C} 8$ & $0.0368(12)$ & $0.0597(17)$ & $0.0487(14)$ & $-0.0210(12)$ & $0.0099(11)$ & -0.0315 \\
\hline C9 & $0.0509(15)$ & $0.0574(18)$ & $0.0607(17)$ & $-0.0311(14)$ & $0.0095(13)$ & $-0.0259(14)$ \\
\hline $\mathrm{C} 10$ & $0.0678(18)$ & $0.0410(15)$ & $0.0591(17)$ & $-0.0207(14)$ & $0.0046(14)$ & $-0.0170(13)$ \\
\hline C11 & $0.0455(13)$ & $0.0433(14)$ & $0.0507(15)$ & $-0.0128(11)$ & 0.0008 (11) & $-0.0186(12)$ \\
\hline $\mathrm{C} 12$ & $0.0300(10)$ & $0.0463(14)$ & $0.0334(11)$ & $-0.0120(10)$ & $0.0046(9)$ & $-0.0221(10)$ \\
\hline $\mathrm{C} 13$ & $0.0298(10)$ & $0.0337(12)$ & $0.0373(12)$ & $-0.0033(9)$ & $-0.0034(9)$ & $-0.0178(10)$ \\
\hline C14 & $0.0396(12)$ & $0.0439(14)$ & $0.0473(14)$ & $-0.0132(11)$ & $0.0116(11)$ & $-0.0217(12)$ \\
\hline $\mathrm{C} 15$ & $0.0525(15)$ & $0.0464(16)$ & $0.0701(19)$ & $-0.0239(13)$ & $0.0183(14)$ & $-0.0245(14)$ \\
\hline $\mathrm{C} 16$ & $0.0493(14)$ & $0.0397(14)$ & $0.078(2)$ & $-0.0167(12)$ & $0.0029(14)$ & $-0.0287(14)$ \\
\hline $\mathrm{C} 17$ & $0.0383(12)$ & $0.0397(13)$ & $0.0556(15)$ & $-0.0055(10)$ & $-0.0061(11)$ & $-0.0272(12)$ \\
\hline $\mathrm{C} 18$ & $0.0564(15)$ & 0.0518 (17) & $0.0673(19)$ & $-0.0064(13)$ & $-0.0096(14)$ & $-0.0413(15)$ \\
\hline C19 & $0.0628(16)$ & 0.0637 (19) & $0.0514(16)$ & $0.0021(14)$ & $-0.0078(13)$ & $-0.0426(15)$ \\
\hline $\mathrm{C} 20$ & $0.0448(12)$ & $0.0485(15)$ & $0.0347(12)$ & $0.0034(11)$ & $-0.0042(10)$ & $-0.0229(11)$ \\
\hline $\mathrm{C} 21$ & $0.0577(15)$ & $0.0673(19)$ & $0.0321(13)$ & $0.0035(14)$ & $0.0048(11)$ & $-0.0235(13)$ \\
\hline $\mathrm{C} 22$ & $0.0520(15)$ & $0.0549(17)$ & $0.0351(13)$ & $-0.0078(13)$ & $0.0126(11)$ & $-0.0108(12)$ \\
\hline $\mathrm{C} 23$ & $0.0424(12)$ & $0.0421(14)$ & $0.0358(13)$ & $-0.0077(11)$ & $0.0062(10)$ & $-0.0119(11)$ \\
\hline $\mathrm{C} 24$ & $0.0324(10)$ & $0.0346(12)$ & $0.0303(11)$ & $0.0019(9)$ & $-0.0046(9)$ & $-0.0156(9)$ \\
\hline $\mathrm{O} 1$ & $0.171(8)$ & $0.152(8)$ & $0.109(6)$ & $-0.028(6)$ & $0.017(5)$ & $-0.050(5)$ \\
\hline $\mathrm{C} 25$ & $0.202(9)$ & $0.147(8)$ & $0.135(8)$ & $-0.061(7)$ & $0.015(7)$ & $-0.069(7)$ \\
\hline $\mathrm{C} 26$ & $0.142(7)$ & $0.100(6)$ & $0.083(5)$ & $-0.003(5)$ & $-0.017(5)$ & $-0.049(5)$ \\
\hline $\mathrm{C} 27$ & $0.172(8)$ & $0.157(8)$ & $0.094(6)$ & $-0.049(6)$ & $0.027(6)$ & $-0.054(6)$ \\
\hline $\mathrm{O} 1^{\prime}$ & $0.147(7)$ & $0.097(6)$ & $0.107(7)$ & $-0.089(5)$ & $0.063(6)$ & $-0.030(4)$ \\
\hline $\mathrm{C} 25^{\prime}$ & $0.139(8)$ & $0.088(6)$ & $0.095(7)$ & $-0.033(6)$ & $0.029(6)$ & $-0.058(5)$ \\
\hline $\mathrm{C} 26^{\prime}$ & $0.144(8)$ & $0.169(10)$ & $0.094(7)$ & $-0.052(7)$ & $0.020(6)$ & $-0.046(7)$ \\
\hline $\mathrm{C} 27^{\prime}$ & $0.149(9)$ & $0.152(10)$ & $0.203(12)$ & $-0.045(8)$ & $0.008(8)$ & $-0.059(8)$ \\
\hline
\end{tabular}

Geometric parameters $\left(\AA,{ }^{\circ}\right)$

\begin{tabular}{llll}
\hline $\mathrm{Zn} 1-\mathrm{N} 1$ & $2.190(2)$ & $\mathrm{C} 10-\mathrm{C} 11$ & $1.399(3)$ \\
$\mathrm{Zn} 1-\mathrm{N} 2$ & $2.198(2)$ & $\mathrm{C} 10-\mathrm{H} 10$ & 0.9300 \\
$\mathrm{Zn} 1-\mathrm{N} 3$ & $2.157(2)$ & $\mathrm{C} 11-\mathrm{H} 11$ & 0.9300 \\
$\mathrm{Zn} 1-\mathrm{N} 4$ & $2.275(2)$ & $\mathrm{C} 13-\mathrm{C} 17$ & $1.407(3)$ \\
$\mathrm{Zn} 1-\mathrm{O} 1 \mathrm{w}$ & $2.090(2)$ & $\mathrm{C} 13-\mathrm{C} 24$ & $1.438(3)$ \\
$\mathrm{Zn} 1-\mathrm{C} 11$ & $2.3520(6)$ & $\mathrm{C} 14-\mathrm{C} 15$ & $1.391(4)$ \\
O1W-H1W1 & $0.85(3)$ & $\mathrm{C} 14-\mathrm{H} 14$ & 0.9300 \\
O1W-H1W2 & $0.84(3)$ & $\mathrm{C} 15-\mathrm{C} 16$ & $1.369(4)$
\end{tabular}




\begin{tabular}{|c|c|c|c|}
\hline $\mathrm{N} 1-\mathrm{C} 2$ & $1.319(3)$ & C15-H15 & 0.9300 \\
\hline $\mathrm{N} 1-\mathrm{C} 1$ & $1.360(3)$ & $\mathrm{C} 16-\mathrm{C} 17$ & $1.400(4)$ \\
\hline $\mathrm{N} 2-\mathrm{C} 11$ & $1.322(3)$ & $\mathrm{C} 16-\mathrm{H} 16$ & 0.9300 \\
\hline $\mathrm{N} 2-\mathrm{C} 12$ & $1.350(3)$ & $\mathrm{C} 17-\mathrm{C} 18$ & $1.431(4)$ \\
\hline $\mathrm{N} 3-\mathrm{C} 14$ & $1.329(3)$ & $\mathrm{C} 18-\mathrm{C} 19$ & $1.349(4)$ \\
\hline $\mathrm{N} 3-\mathrm{C} 13$ & $1.355(3)$ & C18-H18 & 0.9300 \\
\hline $\mathrm{N} 4-\mathrm{C} 23$ & $1.314(3)$ & $\mathrm{C} 19-\mathrm{C} 20$ & $1.423(4)$ \\
\hline $\mathrm{N} 4-\mathrm{C} 24$ & $1.365(3)$ & C19-H19 & 0.9300 \\
\hline N5-C25' & $1.320(10)$ & $\mathrm{C} 20-\mathrm{C} 24$ & $1.404(3)$ \\
\hline N5-C25 & $1.325(11)$ & $\mathrm{C} 20-\mathrm{C} 21$ & $1.416(4)$ \\
\hline $\mathrm{N} 5-\mathrm{C} 26^{\prime}$ & $1.424(8)$ & $\mathrm{C} 21-\mathrm{C} 22$ & $1.354(4)$ \\
\hline N5-C26 & $1.431(7)$ & $\mathrm{C} 21-\mathrm{H} 21$ & 0.9300 \\
\hline $\mathrm{N} 5-\mathrm{C} 27^{\prime}$ & $1.432(9)$ & $\mathrm{C} 22-\mathrm{C} 23$ & $1.397(4)$ \\
\hline N5-C27 & $1.487(7)$ & $\mathrm{C} 22-\mathrm{H} 22$ & 0.9300 \\
\hline $\mathrm{C} 1-\mathrm{C} 5$ & $1.410(3)$ & $\mathrm{C} 23-\mathrm{H} 23$ & 0.9300 \\
\hline $\mathrm{C} 1-\mathrm{C} 12$ & $1.435(3)$ & $\mathrm{O} 1-\mathrm{C} 25$ & $1.242(9)$ \\
\hline $\mathrm{C} 2-\mathrm{C} 3$ & $1.397(3)$ & $\mathrm{C} 25-\mathrm{H} 25$ & 0.9300 \\
\hline $\mathrm{C} 2-\mathrm{H} 2$ & 0.9300 & $\mathrm{C} 26-\mathrm{H} 26 \mathrm{~A}$ & 0.9600 \\
\hline $\mathrm{C} 3-\mathrm{C} 4$ & $1.357(4)$ & $\mathrm{C} 26-\mathrm{H} 26 \mathrm{~B}$ & 0.9600 \\
\hline $\mathrm{C} 3-\mathrm{H} 3$ & 0.9300 & $\mathrm{C} 26-\mathrm{H} 26 \mathrm{C}$ & 0.9600 \\
\hline $\mathrm{C} 4-\mathrm{C} 5$ & $1.401(4)$ & $\mathrm{C} 27-\mathrm{H} 27 \mathrm{~A}$ & 0.9600 \\
\hline $\mathrm{C} 4-\mathrm{H} 4$ & 0.9300 & $\mathrm{C} 27-\mathrm{H} 27 \mathrm{~B}$ & 0.9600 \\
\hline $\mathrm{C} 5-\mathrm{C} 6$ & $1.433(4)$ & $\mathrm{C} 27-\mathrm{H} 27 \mathrm{C}$ & 0.9600 \\
\hline $\mathrm{C} 6-\mathrm{C} 7$ & $1.332(4)$ & $\mathrm{O} 1^{\prime}-\mathrm{C} 25^{\prime}$ & $1.291(19)$ \\
\hline $\mathrm{C} 6-\mathrm{H} 6$ & 0.9300 & $\mathrm{C} 25^{\prime}-\mathrm{H} 25^{\prime}$ & 0.9300 \\
\hline $\mathrm{C} 7-\mathrm{C} 8$ & $1.428(4)$ & $\mathrm{C} 26^{\prime}-\mathrm{H} 26 \mathrm{D}$ & 0.9600 \\
\hline $\mathrm{C} 7-\mathrm{H} 7$ & 0.9300 & $\mathrm{C} 26^{\prime}-\mathrm{H} 26 \mathrm{E}$ & 0.9600 \\
\hline $\mathrm{C} 8-\mathrm{C} 9$ & $1.408(4)$ & $\mathrm{C} 26^{\prime}-\mathrm{H} 26 \mathrm{~F}$ & 0.9600 \\
\hline $\mathrm{C} 8-\mathrm{C} 12$ & $1.414(3)$ & $\mathrm{C} 27^{\prime}-\mathrm{H} 27 \mathrm{D}$ & 0.9600 \\
\hline $\mathrm{C} 9-\mathrm{C} 10$ & $1.357(4)$ & $\mathrm{C} 27^{\prime}-\mathrm{H} 27 \mathrm{E}$ & 0.9600 \\
\hline C9-H9 & 0.9300 & $\mathrm{C} 27^{\prime}-\mathrm{H} 27 \mathrm{~F}$ & 0.9600 \\
\hline $\mathrm{O} 1 \mathrm{~W}-\mathrm{Zn} 1-\mathrm{N} 3$ & $96.59(7)$ & $\mathrm{N} 2-\mathrm{C} 11-\mathrm{C} 10$ & $122.9(2)$ \\
\hline $\mathrm{O} 1 \mathrm{~W}-\mathrm{Zn} 1-\mathrm{N} 1$ & $167.57(6)$ & $\mathrm{N} 2-\mathrm{C} 11-\mathrm{H} 11$ & 118.5 \\
\hline N3-Zn1-N1 & $91.38(7)$ & $\mathrm{C} 10-\mathrm{C} 11-\mathrm{H} 11$ & 118.5 \\
\hline $\mathrm{O} 1 \mathrm{~W}-\mathrm{Zn} 1-\mathrm{N} 2$ & $93.77(7)$ & $\mathrm{N} 2-\mathrm{C} 12-\mathrm{C} 8$ & $123.0(2)$ \\
\hline $\mathrm{N} 3-\mathrm{Zn} 1-\mathrm{N} 2$ & $160.59(7)$ & $\mathrm{N} 2-\mathrm{C} 12-\mathrm{C} 1$ & 118.07 (19) \\
\hline $\mathrm{N} 1-\mathrm{Zn} 1-\mathrm{N} 2$ & $75.98(7)$ & $\mathrm{C} 8-\mathrm{C} 12-\mathrm{C} 1$ & $118.9(2)$ \\
\hline $\mathrm{O} 1 \mathrm{~W}-\mathrm{Zn} 1-\mathrm{N} 4$ & $86.13(7)$ & $\mathrm{N} 3-\mathrm{C} 13-\mathrm{C} 17$ & $122.6(2)$ \\
\hline N3-Zn1-N4 & $75.15(6)$ & $\mathrm{N} 3-\mathrm{C} 13-\mathrm{C} 24$ & $117.74(19)$ \\
\hline $\mathrm{N} 1-\mathrm{Zn} 1-\mathrm{N} 4$ & $86.72(6)$ & $\mathrm{C} 17-\mathrm{C} 13-\mathrm{C} 24$ & $119.6(2)$ \\
\hline $\mathrm{N} 2-\mathrm{Zn} 1-\mathrm{N} 4$ & $89.27(7)$ & $\mathrm{N} 3-\mathrm{C} 14-\mathrm{C} 15$ & $123.4(2)$ \\
\hline $\mathrm{O} 1 \mathrm{~W}-\mathrm{Zn} 1-\mathrm{Cl} 1$ & $93.08(5)$ & $\mathrm{N} 3-\mathrm{C} 14-\mathrm{H} 14$ & 118.3 \\
\hline N3-Zn1-Cl1 & $97.30(5)$ & $\mathrm{C} 15-\mathrm{C} 14-\mathrm{H} 14$ & 118.3 \\
\hline $\mathrm{N} 1-\mathrm{Zn} 1-\mathrm{Cl} 1$ & $95.36(5)$ & $\mathrm{C} 16-\mathrm{C} 15-\mathrm{C} 14$ & $119.0(2)$ \\
\hline $\mathrm{N} 2-\mathrm{Zn} 1-\mathrm{Cl} 1$ & $98.48(5)$ & $\mathrm{C} 16-\mathrm{C} 15-\mathrm{H} 15$ & 120.5 \\
\hline $\mathrm{N} 4-\mathrm{Zn} 1-\mathrm{Cl1}$ & $172.25(5)$ & $\mathrm{C} 14-\mathrm{C} 15-\mathrm{H} 15$ & 120.5 \\
\hline $\mathrm{Zn} 1-\mathrm{O} 1 \mathrm{~W}-\mathrm{H} 1 \mathrm{~W} 1$ & $114(2)$ & $\mathrm{C} 15-\mathrm{C} 16-\mathrm{C} 17$ & $119.6(2)$ \\
\hline
\end{tabular}




\begin{tabular}{|c|c|c|c|}
\hline $\mathrm{Zn} 1-\mathrm{O} 1 \mathrm{~W}-\mathrm{H} 1 \mathrm{~W} 2$ & $120(2)$ & $\mathrm{C} 15-\mathrm{C} 16-\mathrm{H} 16$ & 120.2 \\
\hline $\mathrm{H} 1 \mathrm{~W} 1-\mathrm{O} 1 \mathrm{~W}-\mathrm{H} 1 \mathrm{~W} 2$ & $110(3)$ & $\mathrm{C} 17-\mathrm{C} 16-\mathrm{H} 16$ & 120.2 \\
\hline $\mathrm{C} 2-\mathrm{N} 1-\mathrm{C} 1$ & $118.2(2)$ & $\mathrm{C} 16-\mathrm{C} 17-\mathrm{C} 13$ & $117.6(2)$ \\
\hline $\mathrm{C} 2-\mathrm{N} 1-\mathrm{Zn} 1$ & $127.51(15)$ & $\mathrm{C} 16-\mathrm{C} 17-\mathrm{C} 18$ & $123.1(2)$ \\
\hline $\mathrm{C} 1-\mathrm{N} 1-\mathrm{Zn} 1$ & $114.19(15)$ & $\mathrm{C} 13-\mathrm{C} 17-\mathrm{C} 18$ & $119.3(2)$ \\
\hline $\mathrm{C} 11-\mathrm{N} 2-\mathrm{C} 12$ & $118.1(2)$ & $\mathrm{C} 19-\mathrm{C} 18-\mathrm{C} 17$ & $120.6(2)$ \\
\hline $\mathrm{C} 11-\mathrm{N} 2-\mathrm{Zn} 1$ & $127.78(16)$ & $\mathrm{C} 19-\mathrm{C} 18-\mathrm{H} 18$ & 119.7 \\
\hline $\mathrm{C} 12-\mathrm{N} 2-\mathrm{Zn} 1$ & $114.09(15)$ & $\mathrm{C} 17-\mathrm{C} 18-\mathrm{H} 18$ & 119.7 \\
\hline $\mathrm{C} 14-\mathrm{N} 3-\mathrm{C} 13$ & $117.80(19)$ & $\mathrm{C} 18-\mathrm{C} 19-\mathrm{C} 20$ & $121.6(2)$ \\
\hline $\mathrm{C} 14-\mathrm{N} 3-\mathrm{Zn} 1$ & $125.44(16)$ & $\mathrm{C} 18-\mathrm{C} 19-\mathrm{H} 19$ & 119.2 \\
\hline $\mathrm{C} 13-\mathrm{N} 3-\mathrm{Zn} 1$ & $116.76(14)$ & $\mathrm{C} 20-\mathrm{C} 19-\mathrm{H} 19$ & 119.2 \\
\hline $\mathrm{C} 23-\mathrm{N} 4-\mathrm{C} 24$ & $117.9(2)$ & $\mathrm{C} 24-\mathrm{C} 20-\mathrm{C} 21$ & $116.6(2)$ \\
\hline $\mathrm{C} 23-\mathrm{N} 4-\mathrm{Zn} 1$ & $129.65(17)$ & $\mathrm{C} 24-\mathrm{C} 20-\mathrm{C} 19$ & $119.4(2)$ \\
\hline $\mathrm{C} 24-\mathrm{N} 4-\mathrm{Zn} 1$ & $112.42(13)$ & $\mathrm{C} 21-\mathrm{C} 20-\mathrm{C} 19$ & $124.0(2)$ \\
\hline $\mathrm{C} 25^{\prime}-\mathrm{N} 5-\mathrm{C} 26^{\prime}$ & $115.4(8)$ & $\mathrm{C} 22-\mathrm{C} 21-\mathrm{C} 20$ & $120.2(2)$ \\
\hline $\mathrm{C} 25-\mathrm{N} 5-\mathrm{C} 26$ & $128.7(7)$ & $\mathrm{C} 22-\mathrm{C} 21-\mathrm{H} 21$ & 119.9 \\
\hline $\mathrm{C} 25^{\prime}-\mathrm{N} 5-\mathrm{C} 27^{\prime}$ & $119.3(10)$ & $\mathrm{C} 20-\mathrm{C} 21-\mathrm{H} 21$ & 119.9 \\
\hline $\mathrm{C} 26^{\prime}-\mathrm{N} 5-\mathrm{C} 27^{\prime}$ & $125.4(9)$ & $\mathrm{C} 21-\mathrm{C} 22-\mathrm{C} 23$ & $118.9(2)$ \\
\hline $\mathrm{C} 25-\mathrm{N} 5-\mathrm{C} 27$ & $119.5(8)$ & $\mathrm{C} 21-\mathrm{C} 22-\mathrm{H} 22$ & 120.6 \\
\hline $\mathrm{C} 26-\mathrm{N} 5-\mathrm{C} 27$ & $111.8(6)$ & $\mathrm{C} 23-\mathrm{C} 22-\mathrm{H} 22$ & 120.6 \\
\hline $\mathrm{N} 1-\mathrm{C} 1-\mathrm{C} 5$ & $122.3(2)$ & $\mathrm{N} 4-\mathrm{C} 23-\mathrm{C} 22$ & $123.6(2)$ \\
\hline $\mathrm{N} 1-\mathrm{C} 1-\mathrm{C} 12$ & $117.64(19)$ & $\mathrm{N} 4-\mathrm{C} 23-\mathrm{H} 23$ & 118.2 \\
\hline $\mathrm{C} 5-\mathrm{C} 1-\mathrm{C} 12$ & $120.0(2)$ & $\mathrm{C} 22-\mathrm{C} 23-\mathrm{H} 23$ & 118.2 \\
\hline $\mathrm{N} 1-\mathrm{C} 2-\mathrm{C} 3$ & $122.9(2)$ & $\mathrm{N} 4-\mathrm{C} 24-\mathrm{C} 20$ & $122.8(2)$ \\
\hline $\mathrm{N} 1-\mathrm{C} 2-\mathrm{H} 2$ & 118.6 & $\mathrm{~N} 4-\mathrm{C} 24-\mathrm{C} 13$ & $117.76(19)$ \\
\hline $\mathrm{C} 3-\mathrm{C} 2-\mathrm{H} 2$ & 118.6 & $\mathrm{C} 20-\mathrm{C} 24-\mathrm{C} 13$ & $119.4(2)$ \\
\hline $\mathrm{C} 4-\mathrm{C} 3-\mathrm{C} 2$ & $119.5(3)$ & $\mathrm{O} 1-\mathrm{C} 25-\mathrm{N} 5$ & $118.4(13)$ \\
\hline $\mathrm{C} 4-\mathrm{C} 3-\mathrm{H} 3$ & 120.2 & $\mathrm{O} 1-\mathrm{C} 25-\mathrm{H} 25$ & 120.8 \\
\hline $\mathrm{C} 2-\mathrm{C} 3-\mathrm{H} 3$ & 120.2 & N5-C25-H25 & 120.8 \\
\hline $\mathrm{C} 3-\mathrm{C} 4-\mathrm{C} 5$ & $119.6(2)$ & $\mathrm{N} 5-\mathrm{C} 26-\mathrm{H} 26 \mathrm{~A}$ & 109.5 \\
\hline $\mathrm{C} 3-\mathrm{C} 4-\mathrm{H} 4$ & 120.2 & $\mathrm{~N} 5-\mathrm{C} 26-\mathrm{H} 26 \mathrm{~B}$ & 109.5 \\
\hline $\mathrm{C} 5-\mathrm{C} 4-\mathrm{H} 4$ & 120.2 & $\mathrm{~N} 5-\mathrm{C} 26-\mathrm{H} 26 \mathrm{C}$ & 109.5 \\
\hline $\mathrm{C} 4-\mathrm{C} 5-\mathrm{C} 1$ & $117.3(2)$ & $\mathrm{N} 5-\mathrm{C} 27-\mathrm{H} 27 \mathrm{~A}$ & 109.5 \\
\hline $\mathrm{C} 4-\mathrm{C} 5-\mathrm{C} 6$ & $123.9(2)$ & $\mathrm{N} 5-\mathrm{C} 27-\mathrm{H} 27 \mathrm{~B}$ & 109.5 \\
\hline $\mathrm{C} 1-\mathrm{C} 5-\mathrm{C} 6$ & $118.8(2)$ & $\mathrm{N} 5-\mathrm{C} 27-\mathrm{H} 27 \mathrm{C}$ & 109.5 \\
\hline $\mathrm{C} 7-\mathrm{C} 6-\mathrm{C} 5$ & $121.4(2)$ & $\mathrm{O} 1^{\prime}-\mathrm{C} 25^{\prime}-\mathrm{N} 5$ & $122.1(11)$ \\
\hline $\mathrm{C} 7-\mathrm{C} 6-\mathrm{H} 6$ & 119.3 & $\mathrm{O} 1^{\prime}-\mathrm{C} 25^{\prime}-\mathrm{H} 25^{\prime}$ & 118.9 \\
\hline $\mathrm{C} 5-\mathrm{C} 6-\mathrm{H} 6$ & 119.3 & $\mathrm{~N} 5-\mathrm{C} 25^{\prime}-\mathrm{H} 25^{\prime}$ & 118.9 \\
\hline $\mathrm{C} 6-\mathrm{C} 7-\mathrm{C} 8$ & $121.4(2)$ & $\mathrm{N} 5-\mathrm{C} 26^{\prime}-\mathrm{H} 26 \mathrm{D}$ & 109.5 \\
\hline $\mathrm{C} 6-\mathrm{C} 7-\mathrm{H} 7$ & 119.3 & $\mathrm{~N} 5-\mathrm{C} 26^{\prime}-\mathrm{H} 26 \mathrm{E}$ & 109.5 \\
\hline $\mathrm{C} 8-\mathrm{C} 7-\mathrm{H} 7$ & 119.3 & $\mathrm{H} 26 \mathrm{D}-\mathrm{C} 26^{\prime}-\mathrm{H} 26 \mathrm{E}$ & 109.5 \\
\hline $\mathrm{C} 9-\mathrm{C} 8-\mathrm{C} 12$ & $116.8(2)$ & $\mathrm{N} 5-\mathrm{C} 26^{\prime}-\mathrm{H} 26 \mathrm{~F}$ & 109.5 \\
\hline $\mathrm{C} 9-\mathrm{C} 8-\mathrm{C} 7$ & $123.9(2)$ & $\mathrm{H} 26 \mathrm{D}-\mathrm{C} 26^{\prime}-\mathrm{H} 26 \mathrm{~F}$ & 109.5 \\
\hline $\mathrm{C} 12-\mathrm{C} 8-\mathrm{C} 7$ & $119.4(3)$ & $\mathrm{H} 26 \mathrm{E}-\mathrm{C} 26^{\prime}-\mathrm{H} 26 \mathrm{~F}$ & 109.5 \\
\hline $\mathrm{C} 10-\mathrm{C} 9-\mathrm{C} 8$ & $119.7(2)$ & $\mathrm{N} 5-\mathrm{C} 27^{\prime}-\mathrm{H} 27 \mathrm{D}$ & 109.5 \\
\hline $\mathrm{C} 10-\mathrm{C} 9-\mathrm{H} 9$ & 120.1 & $\mathrm{~N} 5-\mathrm{C} 27^{\prime}-\mathrm{H} 27 \mathrm{E}$ & 109.5 \\
\hline $\mathrm{C} 8-\mathrm{C} 9-\mathrm{H} 9$ & 120.1 & $\mathrm{H} 27 \mathrm{D}-\mathrm{C} 27^{\prime}-\mathrm{H} 27 \mathrm{E}$ & 109.5 \\
\hline $\mathrm{C} 9-\mathrm{C} 10-\mathrm{C} 11$ & $119.4(3)$ & $\mathrm{N} 5-\mathrm{C} 27^{\prime}-\mathrm{H} 27 \mathrm{~F}$ & 109.5 \\
\hline
\end{tabular}




\begin{tabular}{|c|c|c|c|}
\hline $\mathrm{C} 9-\mathrm{C} 10-\mathrm{H} 10$ & 120.3 & $\mathrm{H} 27 \mathrm{D}-\mathrm{C} 27^{\prime}-\mathrm{H} 27 \mathrm{~F}$ & 109.5 \\
\hline $\mathrm{C} 11-\mathrm{C} 10-\mathrm{H} 10$ & 120.3 & $\mathrm{H} 27 \mathrm{E}-\mathrm{C} 27^{\prime}-\mathrm{H} 27 \mathrm{~F}$ & 109.5 \\
\hline $\mathrm{O} 1 \mathrm{~W}-\mathrm{Zn} 1-\mathrm{N} 1-\mathrm{C} 2$ & $-146.2(3)$ & $\mathrm{C} 7-\mathrm{C} 8-\mathrm{C} 9-\mathrm{C} 10$ & $-178.8(3)$ \\
\hline $\mathrm{N} 3-\mathrm{Zn} 1-\mathrm{N} 1-\mathrm{C} 2$ & $-16.20(19)$ & $\mathrm{C} 8-\mathrm{C} 9-\mathrm{C} 10-\mathrm{C} 11$ & $-1.1(4)$ \\
\hline $\mathrm{N} 2-\mathrm{Zn} 1-\mathrm{N} 1-\mathrm{C} 2$ & $178.7(2)$ & $\mathrm{C} 12-\mathrm{N} 2-\mathrm{C} 11-\mathrm{C} 10$ & $0.0(4)$ \\
\hline $\mathrm{N} 4-\mathrm{Zn} 1-\mathrm{N} 1-\mathrm{C} 2$ & $-91.24(19)$ & $\mathrm{Zn} 1-\mathrm{N} 2-\mathrm{C} 11-\mathrm{C} 10$ & $178.9(2)$ \\
\hline $\mathrm{C} 11-\mathrm{Zn} 1-\mathrm{N} 1-\mathrm{C} 2$ & $81.27(19)$ & $\mathrm{C} 9-\mathrm{C} 10-\mathrm{C} 11-\mathrm{N} 2$ & $0.6(4)$ \\
\hline $\mathrm{O} 1 \mathrm{~W}-\mathrm{Zn} 1-\mathrm{N} 1-\mathrm{C} 1$ & $36.5(4)$ & $\mathrm{C} 11-\mathrm{N} 2-\mathrm{C} 12-\mathrm{C} 8$ & $-0.2(3)$ \\
\hline $\mathrm{N} 3-\mathrm{Zn} 1-\mathrm{N} 1-\mathrm{C} 1$ & $166.52(14)$ & $\mathrm{Zn} 1-\mathrm{N} 2-\mathrm{C} 12-\mathrm{C} 8$ & $-179.21(17)$ \\
\hline $\mathrm{N} 2-\mathrm{Zn} 1-\mathrm{N} 1-\mathrm{C} 1$ & $1.41(14)$ & $\mathrm{C} 11-\mathrm{N} 2-\mathrm{C} 12-\mathrm{C} 1$ & $179.3(2)$ \\
\hline $\mathrm{N} 4-\mathrm{Zn} 1-\mathrm{N} 1-\mathrm{C} 1$ & $91.48(14)$ & $\mathrm{Zn} 1-\mathrm{N} 2-\mathrm{C} 12-\mathrm{C} 1$ & $0.3(2)$ \\
\hline $\mathrm{C} 11-\mathrm{Zn} 1-\mathrm{N} 1-\mathrm{C} 1$ & $-96.01(14)$ & $\mathrm{C} 9-\mathrm{C} 8-\mathrm{C} 12-\mathrm{N} 2$ & $-0.2(3)$ \\
\hline $\mathrm{O} 1 \mathrm{~W}-\mathrm{Zn} 1-\mathrm{N} 2-\mathrm{C} 11$ & $7.4(2)$ & $\mathrm{C} 7-\mathrm{C} 8-\mathrm{C} 12-\mathrm{N} 2$ & $179.5(2)$ \\
\hline N3-Zn1-N2-C11 & $129.6(2)$ & $\mathrm{C} 9-\mathrm{C} 8-\mathrm{C} 12-\mathrm{C} 1$ & $-179.8(2)$ \\
\hline $\mathrm{N} 1-\mathrm{Zn} 1-\mathrm{N} 2-\mathrm{C} 11$ & $-179.8(2)$ & $\mathrm{C} 7-\mathrm{C} 8-\mathrm{C} 12-\mathrm{C} 1$ & $0.0(3)$ \\
\hline $\mathrm{N} 4-\mathrm{Zn} 1-\mathrm{N} 2-\mathrm{C} 11$ & $93.4(2)$ & $\mathrm{N} 1-\mathrm{C} 1-\mathrm{C} 12-\mathrm{N} 2$ & $1.0(3)$ \\
\hline $\mathrm{Cl1}-\mathrm{Zn} 1-\mathrm{N} 2-\mathrm{C} 11$ & $-86.3(2)$ & $\mathrm{C} 5-\mathrm{C} 1-\mathrm{C} 12-\mathrm{N} 2$ & $-177.85(19)$ \\
\hline $\mathrm{O} 1 \mathrm{~W}-\mathrm{Zn} 1-\mathrm{N} 2-\mathrm{C} 12$ & $-173.77(15)$ & $\mathrm{N} 1-\mathrm{C} 1-\mathrm{C} 12-\mathrm{C} 8$ & $-179.48(19)$ \\
\hline N3-Zn1-N2-C12 & $-51.5(3)$ & $\mathrm{C} 5-\mathrm{C} 1-\mathrm{C} 12-\mathrm{C} 8$ & $1.7(3)$ \\
\hline $\mathrm{N} 1-\mathrm{Zn} 1-\mathrm{N} 2-\mathrm{C} 12$ & $-0.90(15)$ & $\mathrm{C} 14-\mathrm{N} 3-\mathrm{C} 13-\mathrm{C} 17$ & $2.6(3)$ \\
\hline $\mathrm{N} 4-\mathrm{Zn} 1-\mathrm{N} 2-\mathrm{C} 12$ & $-87.70(15)$ & $\mathrm{Zn} 1-\mathrm{N} 3-\mathrm{C} 13-\mathrm{C} 17$ & $-177.17(17)$ \\
\hline $\mathrm{C} 11-\mathrm{Zn} 1-\mathrm{N} 2-\mathrm{C} 12$ & $92.54(15)$ & $\mathrm{C} 14-\mathrm{N} 3-\mathrm{C} 13-\mathrm{C} 24$ & $-176.9(2)$ \\
\hline $\mathrm{O} 1 \mathrm{~W}-\mathrm{Zn} 1-\mathrm{N} 3-\mathrm{C} 14$ & $-99.15(19)$ & $\mathrm{Zn} 1-\mathrm{N} 3-\mathrm{C} 13-\mathrm{C} 24$ & $3.3(3)$ \\
\hline N1-Zn1-N3-C14 & $90.41(19)$ & $\mathrm{C} 13-\mathrm{N} 3-\mathrm{C} 14-\mathrm{C} 15$ & $-2.3(4)$ \\
\hline $\mathrm{N} 2-\mathrm{Zn} 1-\mathrm{N} 3-\mathrm{C} 14$ & $139.0(2)$ & $\mathrm{Zn} 1-\mathrm{N} 3-\mathrm{C} 14-\mathrm{C} 15$ & $177.5(2)$ \\
\hline N4-Zn1-N3-C14 & $176.6(2)$ & $\mathrm{N} 3-\mathrm{C} 14-\mathrm{C} 15-\mathrm{C} 16$ & $0.2(4)$ \\
\hline $\mathrm{C} 11-\mathrm{Zn} 1-\mathrm{N} 3-\mathrm{C} 14$ & $-5.17(19)$ & $\mathrm{C} 14-\mathrm{C} 15-\mathrm{C} 16-\mathrm{C} 17$ & $1.7(4)$ \\
\hline $\mathrm{O} 1 \mathrm{~W}-\mathrm{Zn} 1-\mathrm{N} 3-\mathrm{C} 13$ & $80.64(16)$ & $\mathrm{C} 15-\mathrm{C} 16-\mathrm{C} 17-\mathrm{C} 13$ & $-1.3(4)$ \\
\hline $\mathrm{N} 1-\mathrm{Zn} 1-\mathrm{N} 3-\mathrm{C} 13$ & $-89.80(16)$ & $\mathrm{C} 15-\mathrm{C} 16-\mathrm{C} 17-\mathrm{C} 18$ & $178.0(3)$ \\
\hline $\mathrm{N} 2-\mathrm{Zn} 1-\mathrm{N} 3-\mathrm{C} 13$ & $-41.2(3)$ & N3-C13-C17-C16 & $-0.9(4)$ \\
\hline $\mathrm{N} 4-\mathrm{Zn} 1-\mathrm{N} 3-\mathrm{C} 13$ & $-3.56(15)$ & $\mathrm{C} 24-\mathrm{C} 13-\mathrm{C} 17-\mathrm{C} 16$ & $178.7(2)$ \\
\hline $\mathrm{C} 11-\mathrm{Zn} 1-\mathrm{N} 3-\mathrm{C} 13$ & $174.62(15)$ & $\mathrm{N} 3-\mathrm{C} 13-\mathrm{C} 17-\mathrm{C} 18$ & $179.8(2)$ \\
\hline $\mathrm{O} 1 \mathrm{~W}-\mathrm{Zn} 1-\mathrm{N} 4-\mathrm{C} 23$ & $82.8(2)$ & $\mathrm{C} 24-\mathrm{C} 13-\mathrm{C} 17-\mathrm{C} 18$ & $-0.6(3)$ \\
\hline N3-Zn1-N4-C23 & $-179.3(2)$ & $\mathrm{C} 16-\mathrm{C} 17-\mathrm{C} 18-\mathrm{C} 19$ & $-178.5(3)$ \\
\hline $\mathrm{N} 1-\mathrm{Zn} 1-\mathrm{N} 4-\mathrm{C} 23$ & $-87.0(2)$ & $\mathrm{C} 13-\mathrm{C} 17-\mathrm{C} 18-\mathrm{C} 19$ & $0.8(4)$ \\
\hline $\mathrm{N} 2-\mathrm{Zn} 1-\mathrm{N} 4-\mathrm{C} 23$ & $-11.0(2)$ & $\mathrm{C} 17-\mathrm{C} 18-\mathrm{C} 19-\mathrm{C} 20$ & $-0.2(4)$ \\
\hline $\mathrm{O} 1 \mathrm{~W}-\mathrm{Zn} 1-\mathrm{N} 4-\mathrm{C} 24$ & $-94.44(15)$ & $\mathrm{C} 18-\mathrm{C} 19-\mathrm{C} 20-\mathrm{C} 24$ & $-0.6(4)$ \\
\hline N3-Zn1-N4-C24 & $3.43(14)$ & $\mathrm{C} 18-\mathrm{C} 19-\mathrm{C} 20-\mathrm{C} 21$ & $178.9(3)$ \\
\hline $\mathrm{N} 1-\mathrm{Zn} 1-\mathrm{N} 4-\mathrm{C} 24$ & $95.73(15)$ & $\mathrm{C} 24-\mathrm{C} 20-\mathrm{C} 21-\mathrm{C} 22$ & $-0.8(4)$ \\
\hline $\mathrm{N} 2-\mathrm{Zn} 1-\mathrm{N} 4-\mathrm{C} 24$ & $171.73(15)$ & $\mathrm{C} 19-\mathrm{C} 20-\mathrm{C} 21-\mathrm{C} 22$ & $179.7(3)$ \\
\hline $\mathrm{C} 2-\mathrm{N} 1-\mathrm{C} 1-\mathrm{C} 5$ & $-0.5(3)$ & $\mathrm{C} 20-\mathrm{C} 21-\mathrm{C} 22-\mathrm{C} 23$ & $0.4(4)$ \\
\hline $\mathrm{Zn} 1-\mathrm{N} 1-\mathrm{C} 1-\mathrm{C} 5$ & $177.05(16)$ & $\mathrm{C} 24-\mathrm{N} 4-\mathrm{C} 23-\mathrm{C} 22$ & $-0.4(4)$ \\
\hline $\mathrm{C} 2-\mathrm{N} 1-\mathrm{C} 1-\mathrm{C} 12$ & $-179.31(19)$ & $\mathrm{Zn} 1-\mathrm{N} 4-\mathrm{C} 23-\mathrm{C} 22$ & $-177.58(18)$ \\
\hline $\mathrm{Zn} 1-\mathrm{N} 1-\mathrm{C} 1-\mathrm{C} 12$ & $-1.8(2)$ & $\mathrm{C} 21-\mathrm{C} 22-\mathrm{C} 23-\mathrm{N} 4$ & $0.3(4)$ \\
\hline $\mathrm{C} 1-\mathrm{N} 1-\mathrm{C} 2-\mathrm{C} 3$ & $1.0(3)$ & $\mathrm{C} 23-\mathrm{N} 4-\mathrm{C} 24-\mathrm{C} 20$ & $0.0(3)$ \\
\hline $\mathrm{Zn} 1-\mathrm{N} 1-\mathrm{C} 2-\mathrm{C} 3$ & $-176.18(17)$ & $\mathrm{Zn} 1-\mathrm{N} 4-\mathrm{C} 24-\mathrm{C} 20$ & $177.58(17)$ \\
\hline $\mathrm{N} 1-\mathrm{C} 2-\mathrm{C} 3-\mathrm{C} 4$ & $-0.4(4)$ & $\mathrm{C} 23-\mathrm{N} 4-\mathrm{C} 24-\mathrm{C} 13$ & $179.4(2)$ \\
\hline
\end{tabular}




$\begin{array}{llll}\mathrm{C} 2-\mathrm{C} 3-\mathrm{C} 4-\mathrm{C} 5 & -0.8(4) & \mathrm{Z} 11-\mathrm{N} 4-\mathrm{C} 24-\mathrm{C} 13 & -3.0(2) \\ \mathrm{C} 3-\mathrm{C} 4-\mathrm{C} 5-\mathrm{C} 1 & 1.2(3) & \mathrm{C} 21-\mathrm{C} 20-\mathrm{C} 24-\mathrm{N} 4 & 0.7(3) \\ \mathrm{C} 3-\mathrm{C} 4-\mathrm{C} 5-\mathrm{C} 6 & -179.4(2) & \mathrm{C} 19-\mathrm{C} 20-\mathrm{C} 24-\mathrm{N} 4 & -179.8(2) \\ \mathrm{N} 1-\mathrm{C} 1-\mathrm{C} 5-\mathrm{C} 4 & -0.6(3) & \mathrm{C} 21-\mathrm{C} 20-\mathrm{C} 24-\mathrm{C} 13 & -178.7(2) \\ \mathrm{C} 12-\mathrm{C} 1-\mathrm{C} 5-\mathrm{C} 4 & 178.2(2) & \mathrm{C} 19-\mathrm{C} 20-\mathrm{C} 24-\mathrm{C} 13 & 0.8(3) \\ \mathrm{N} 1-\mathrm{C} 1-\mathrm{C} 5-\mathrm{C} 6 & -180.0(2) & \mathrm{N} 3-\mathrm{C} 13-\mathrm{C} 24-\mathrm{N} 4 & 0.0(3) \\ \mathrm{C} 12-\mathrm{C} 1-\mathrm{C} 5-\mathrm{C} 6 & -1.2(3) & \mathrm{C} 17-\mathrm{C} 13-\mathrm{C} 24-\mathrm{N} 4 & -179.6(2) \\ \mathrm{C} 4-\mathrm{C} 5-\mathrm{C} 6-\mathrm{C} 7 & 179.6(2) & \mathrm{N} 3-\mathrm{C} 13-\mathrm{C} 24-\mathrm{C} 20 & 179.4(2) \\ \mathrm{C} 1-\mathrm{C} 5-\mathrm{C} 6-\mathrm{C} 7 & -1.1(4) & \mathrm{C} 17-\mathrm{C} 13-\mathrm{C} 24-\mathrm{C} 20 & -0.1(3) \\ \mathrm{C} 5-\mathrm{C} 6-\mathrm{C} 7-\mathrm{C} 8 & 2.9(4) & \mathrm{C} 26-\mathrm{N} 5-\mathrm{C} 25-\mathrm{O} 1 & 0.8(4) \\ \mathrm{C} 6-\mathrm{C} 7-\mathrm{C} 8-\mathrm{C} 9 & 177.4(3) & \mathrm{C} 27-\mathrm{N} 5-\mathrm{C} 25-\mathrm{O} 1 & 179.3(3) \\ \mathrm{C} 6-\mathrm{C} 7-\mathrm{C} 8-\mathrm{C} 12 & -2.3(4) & \mathrm{C} 26^{\prime}-\mathrm{N} 5-\mathrm{C} 25^{\prime}-\mathrm{O} 1^{\prime} & 0.8(3) \\ \mathrm{C} 12-\mathrm{C} 8-\mathrm{C} 9-\mathrm{C} 10 & 0.9(4) & \mathrm{C} 27^{\prime}-\mathrm{N} 5-\mathrm{C} 25^{\prime}-\mathrm{O} 1^{\prime} & -179.9(3)\end{array}$

Hydrogen-bond geometry $\left(\AA,{ }^{\circ}\right)$

\begin{tabular}{lllll}
\hline$D-\mathrm{H} \cdots A$ & $D-\mathrm{H}$ & $\mathrm{H} \cdots A$ & $D \cdots A$ & $D-\mathrm{H} \cdots A$ \\
\hline $\mathrm{O} 1 \mathrm{w}-\mathrm{H} 1 \mathrm{w} 1 \cdots \mathrm{Cl} 2$ & $0.85(3)$ & $2.29(3)$ & $3.112(2)$ & $163(3)$ \\
$\mathrm{O} 1 \mathrm{w}-\mathrm{H} 1 \mathrm{w} 2 \cdots \mathrm{C} 2^{\mathrm{i}}$ & $0.84(3)$ & $2.24(3)$ & $3.079(2)$ & $172(3)$ \\
\hline
\end{tabular}

Symmetry code: (i) $-x+1,-y+2,-z+1$. 